\title{
L'alternance des voyelles moyennes en coratin: une analyse basée sur la théorie des éléments
}

\author{
JONATHAN BUCCI \\ Université Côte d'Azur, CNRS, Bases Corpus Langage (BCL) et Université \\ Grenoble Alpes \\ buccijo@gmail.com
}

\begin{abstract}
Résumé
Le coratin est un dialecte parlé dans la région des Pouilles où l'on observe un processus de réduction vocalique : toutes les voyelles atones se réduisent à schwa sauf /a/. Cependant, cette réduction n'a pas lieu lorsqu'elles sont adjacentes à une consonne qui partage de la mélodie comme la palatalité pour les voyelles antérieures et la vélarité ou la labialité pour les voyelles postérieures. On observe également que l'ATRité des voyelles moyennes alterne : / $/ \mathcal{\varepsilon}, \mathrm{J}$ tonique se réalisent $[\mathrm{e}, \mathrm{o}]$ en position atone et /o,o/ font surface en position atone par un $[\mathrm{u}]$ uniquement lorsque la consonne adjacente est vélaire. L'analyse de cette alternance sera basée sur une version de la Théorie des Eléments ainsi que sur des contraintes de licenciement qui définissent d'une part le choix particulier qu'une langue fait des possibilités combinatoires, et d'autre part participe à définir les propriétés des processus phonologiques de la langue.
\end{abstract}

Mots-clés: réduction vocalique, phonologie, dialectologie, longueur virtuelle, théorie des éléments

\begin{abstract}
Coratino is a dialect spoken in the region of Puglia, in which there is a process of vowel reduction: all unstressed vowels except /a/ are reduced to schwa. However, this reduction does not happen when a vowel is adjacent to a consonant that shares a melody element, such as palatality for front vowels and velarity or labiality for back vowels. In addition, the ATR property of the mid vowels alternates: stressed $/ \varepsilon, \mathrm{o} /$ are realized as $[\mathrm{e}, \mathrm{o}]$ in unstressed positions, and /o,o/ surface as $[\mathrm{u}]$ only when the adjacent consonant is velar. The analysis of this alternation is based on a version of Element Theory, and also on the licensing constraints that on the one hand define the specific choice a language makes among the combinatory possibilities, and on the other hand play a role in defining the properties of the phonological processes in the language.
\end{abstract}

Keywords: vowel reduction, phonology, dialectology, virtual length, element theory

Je remercie Tobias Scheer, Elisabetta Carpitelli et les trois évaluateurs anonymes pour les commentaires, les discussions et les relectures qu'ils ont consacrés à ce manuscrit. Je souhaiterais également rendre hommage à mon père (qui était un de mes informateurs coratins) malheureusement décédé pendant la soumission de ce manuscrit. 


\section{INTRODUCTION}

Cet article a pour objectif de décrire et d'analyser un processus phonologique où l'ATRité $^{1}$ des voyelles moyennes alterne dans le dialecte italo-roman de Corato. ${ }^{2}$ En effet, les voyelles $/ \varepsilon, \mathrm{J} /$ toniques se réalisent $[\mathrm{e}, \mathrm{o}]$ en position atone et $/ \mathrm{o} /$ tonique fait surface en tant que [u] dans le même contexte lorsqu'il est adjacent à une consonne vélaire.

Cette étude s'appuie sur l'analyse de la réduction vocalique ${ }^{3}$ vue dans Bucci (2013a, 2013b) qui a aussi été décrite dans un autre cadre ${ }^{4}$ (D'Introno et Weston 1997, 2000). Ce processus de réduction vocalique consiste en la réduction à schwa des voyelles périphériques atones $/ \mathrm{i}, \mathrm{e}, \varepsilon, \mathrm{\jmath}, \mathrm{o}, \mathrm{u} / \mathrm{sauf} / \mathrm{a} /$. De manière inattendue, les voyelles atones postérieures $/ \mathrm{o}, \mathrm{u} /$ et antérieures $/ \mathrm{i}, \mathrm{e} /$ résistent à la réduction lorsqu'elles sont en position tonique, adjacentes à une consonne qui partage le lieu d'articulation, c'est-à-dire soit la labialité/vélarité pour les voyelles /o,u/, soit la palatalité pour les voyelles /i,e/. Cette idée que le partage de traits mélodiques (matérialisé par une structure branchante) confère de la force contre les lénitions a été introduite par Honeybone (2005). Un aperçu de cette analyse sera exposé en section 3; la situation globale des ingrédients théoriques permettant de réduire la disjonction des deux contextes de maintien des voyelles sont :

- l'identification de l'accent tonique en tant qu'espace syllabique (Chierchia 1986, Larsen 1998, Ségéral et Scheer 2008),

- la longueur dite virtuelle (Lowenstamm 1991; Bendjaballah 1995, 2001, 2005).

En section 3.2.1 sera présenté le fonctionnement de l'allongement tonique en italien standard puis, en section 3.2.2, nous verrons le cas des dialectes méridionaux concernant les contraintes métrico-syllabiques pour l'allongement vocalique de ces variétés. Le cas du coratin sera abordé en section 3.2.3 où les données récoltées sur le terrain montreront que le coratin diffère des autres dialectes de cette aire linguistique.

Pour comprendre l'alternance des voyelles moyennes en position atone décrite supra et en section 4, nous nous baserons sur la Théorie des structures internes des segments formulée par Backley (2011) qui sera présentée sous 4.1. Nous verrons en quoi cette Théorie des éléments (ci-après désignée TE) - et uniquement celle-ci, ${ }^{5}$

\footnotetext{
${ }^{1}$ Abréviations : ATR : Advanced Tongue Root feature (avancée de la racine de la langue); AUGM : augmentatif; dent : dentale; DIM : diminutif; lab : labiale; IMP: impératif; nas : nasale; occl : occlusive; TE : Théorie des éléments; vél : vélaire; pal : palatale.

${ }^{2}$ Le coratin est un dialecte de l'aire apulo-barese parlé dans la ville de Corato, située dans la région des Pouilles à environ $40 \mathrm{~km}$ au nord de Bari, en Italie méridionale.

${ }^{3} \mathrm{La}$ réduction vocalique est un processus bien connu dans les dialectes italo-romans méridionaux (Loporcaro 1988 : 57, 2011; Rohlfs 1966-1969).

${ }^{4}$ Les auteurs ont recours à la théorie de sous-spécification introduite par Archangeli (1988) ainsi que l'hypothèse de dépendance (Mester 1986) pour décrire le système du coratin.

${ }^{5}$ Les versions précédentes de la TE comportent certains éléments qui sont différents et, par conséquent, l'utilisation d'une autre version que celle de Backley (2011) forcerait à revoir les contraintes de licenciement. En effet, si on regarde la version de Harris et Lindsey (1995: 70),
} 
par rapport aux autres versions de TE - associée à des contraintes de licenciement paramétrées en coratin (Charette et Göksel 1994, 1996; Kaye 2001 et Scheer 2010) permettra de rendre compte de ces alternances dans ce dialecte. L'analyse sera formalisée dans un cadre théorique neutre en utilisant des points squelettaux $\mathrm{X}$ de type « autosegmental » à la manière de Chierchia (1986).

\section{LE CORATIN : INVENTAIRE SEGMENTAL ET SUFFIXATION}

Le dialecte de Corato, comme tous les dialectes du type apulo-barese, est constitué de 22 consonnes et de sept phonèmes vocaliques : /i,e,, , a,o, $, \mathrm{u}, \mathrm{u} /$ en position tonique (Valente 1975; Stehl 1980, 1988). En revanche, en position atone, le contraste des voyelles est réduit en surface à [a] et [ə], sauf dans les cas de maintien présentés sous (3).

A priori, pour observer l'attitude des voyelles toniques/atones, il a fallu établir des paires de mots (1) où la voyelle du radical était dans un premier temps sous l'effet de l'accent et, dans un second temps, en position non tonique.

Ces paires de mots sont constituées d'un radical en isolation d'une part, et d'autre part d'un radical suffixé. Manifestement, le suffixe est toujours accentué dans ce dialecte, permettant ainsi de saisir le processus de réduction/maintien vocalique et, par conséquent, l'alternance des voyelles moyennes en position atone. Sous (1) sont présentées les paires de mots qui ont servi de base pour la constitution du corpus. $^{6}$

(1) radical

\begin{tabular}{|c|c|c|}
\hline & & \\
\hline ot & 'roue' & rot + ' $\varepsilon$ dd \\
\hline & 'ét & st \\
\hline & 'veste' & dzakk + 'cttə \\
\hline
\end{tabular}

Ayant décrit le système consonantique et vocalique du coratin ainsi que le fonctionnement de l'accent, nous pouvons exposer le processus de réduction/maintien vocalique.

\section{DONNÉES DE LA RÉDUCTION/DU MAINTIEN DES VOYELLES ATONES ET ANALYSE DU PROCESSUS}

Nous avons vu en introduction que toutes les voyelles atones $/ \mathrm{i}, \mathrm{e}, \varepsilon, \mathrm{o}, \mathrm{\jmath}, \mathrm{u} / \mathrm{se}$ réduisent

à schwa en position atone à l'exception du phonème /a/ comme sous (2).

il s'avère que la labialité est représentée par un élément $U$ alors que la vélarité correspond à l'élément @. Cette différence ne permettrait pas de traiter l'alternance des voyelles /o, ว/ se réalisant $[\mathrm{u}]$ dans un contexte de consonne vélaire (cf.18c) avec l'analyse proposée.

${ }^{6}$ Les données présentées dans cet article sont issues de nos enquêtes de terrain et par conséquent sont des données de première main. Le corpus constitué de 264 paires de mots a été réalisé avec l'aide du dictionnaire du coratin de Bucci (1982) puis il a été soumis à des locuteurs natifs à Corato. 
(2) Réduction des voyelles en position atone

a. voyelles postérieures

\begin{tabular}{|c|c|c|c|c|c|}
\hline \multirow{3}{*}{ o : } & & \multicolumn{2}{|l|}{ tonique } & \multicolumn{2}{|l|}{ non-tonique } \\
\hline & /dent_dent & r'otə & 'roue' & $\operatorname{rot}^{\prime} \varepsilon \mathrm{dd} \partial$ & 'roue-DIM' \\
\hline & /dent_dent & d'ormə & 'il dort' & dərm'i & 'dormir' \\
\hline & /dent_dent & s'or ort $\widehat{x}$ & ‘souris’ & sə̄rət $\widehat{\int} \varepsilon \mathrm{cc} \partial$ & 'souris-DIM' \\
\hline & /pal__dent & pānn'ottə & ‘pain' & papnət' ${ }^{\prime} \varepsilon d d ə$ & 'pain-DIM’ \\
\hline & /pal__dent & wann'onə & 'garçon' & wajpə- & 'garçon-DIM' \\
\hline $\mathbf{u}:$ & /dent__pal & abbr' $\underline{\mathbf{u}} \int \partial$ & '(tu) brûles' & abbrə] $\int^{\prime} \mathrm{a}$ & 'brûler' \\
\hline & /dent_pal & fən' & 'fenouil' & fənəccc' $\varepsilon$ dd & 'fenouil-DIM' \\
\hline & /dent__pal & $\mathrm{r}^{\prime} \underline{\mathbf{u}} \hat{\int} \widehat{\partial}$ & 'frit' & ret $\int \partial t^{\prime} a$ & 'frire' \\
\hline & /dent_dent & sfərt'uñ & 'malchance' & sfərtə̄n'attə & 'malchanceux' \\
\hline
\end{tabular}

b. voyelles antérieures

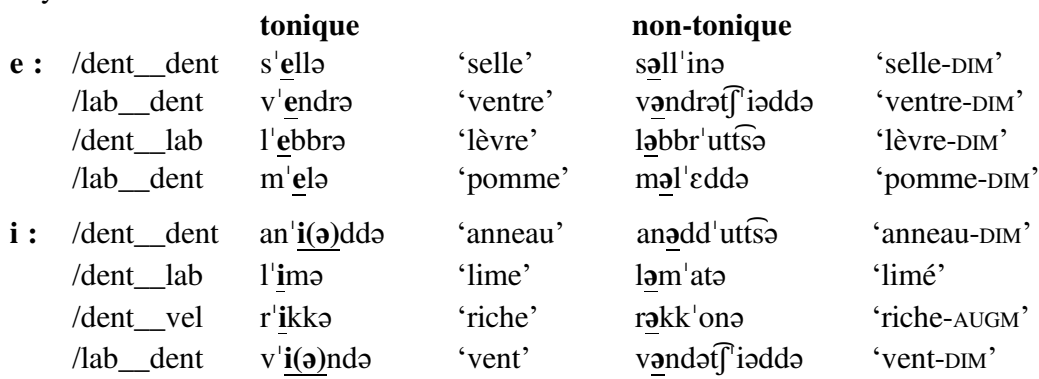

Néanmoins, cette réduction vocalique n'opère pas lorsque les voyelles sont adjacentes à une consonne qui partage leur lieu d'articulation (D'Introno et Weston 1997, 2000; Bucci 2013a, 2013b), c'est-à-dire la labialité, la vélarité (pour les voyelles postérieures) ou la palatalité (pour les voyelles antérieures). Les données sous (3) illustrent le maintien des voyelles en position atone.

(3) Maintien des voyelles en position atone ${ }^{7}$

a. voyelles postérieures :

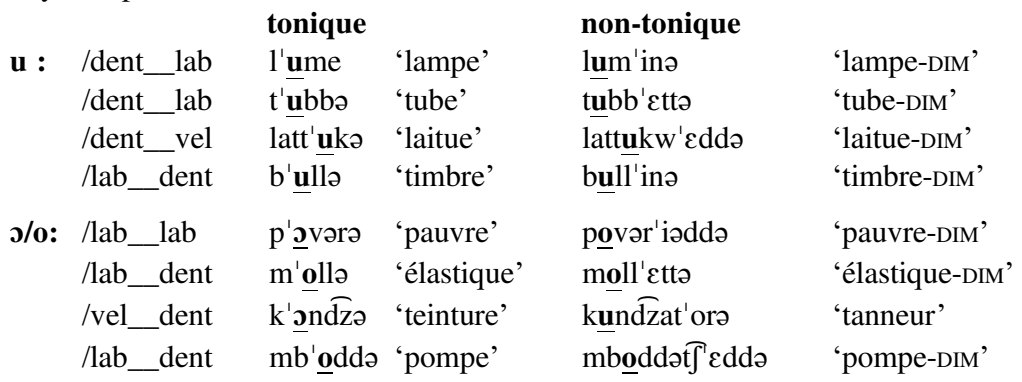

\footnotetext{
${ }^{7}$ Le maintien de la voyelle /a/ a lieu avec n'importe quelle consonne adjacente.
} 
b. voyelles antérieures :

\begin{tabular}{|c|c|c|c|c|}
\hline \multirow{5}{*}{$\begin{array}{l}\text { /dent__pal } \\
\text { /pal__dent } \\
\text { /dent__pal } \\
\text { /dent__pal }\end{array}$} & \multicolumn{2}{|l|}{ tonique } & \multicolumn{2}{|l|}{ non-tonique } \\
\hline & butt' $\underline{\mathbf{e}} \kappa \hat{\gamma}$ & 'bouteille' & butte $\Lambda \Lambda^{\prime}$ 'onə & 'bouteille-DIM' \\
\hline & c'esə & 'église' & cesar' $\varepsilon$ ddə & 'église-DIM' \\
\hline & 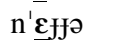 & 'brouillard' & ne-fy'usə & 'brumeux' \\
\hline & s'ecco & 'sceau' & seccot'iddə & 'sceau-DIM' \\
\hline /dent__pal & br'iffə & 'bride' & mbriff'a & 'mettre la bride' \\
\hline /lab__pal & $\mathrm{f}^{\prime} \mathbf{i}$ If于 & 'fille' & fijfy'cttə & 'fille-DIM' \\
\hline /ab_pal & v'inn’ & 'vigne' & vinnət' $\varepsilon$ ddə & 'vigne-DIM' \\
\hline /pal_lab & $\int^{\prime}$ immjə & 'singe' & Jimmj'cttə & 'singe-DIM' \\
\hline
\end{tabular}

Pour récapituler, il y a deux contextes de maintien des voyelles en coratin :

- lorsqu'elles sont toniques,

- lorsqu'elles sont adjacentes à une consonne qui partage un trait articulatoire.

On sait que ces deux contextes produisent le même effet, c'est-à-dire la nonréduction de la voyelle à schwa. Afin de réduire la disjonction de ces contextes, il faut trouver la propriété structurelle commune.

\subsection{Le branchement rend plus fort}

L'analyse de ce processus repose sur l'étude d'Honeybone (2005), qui montre que les structures branchantes résistent aux lénitions. En nous basant sur ce travail, nous avons proposé que dans le cas du coratin, une voyelle atone adjacente à une consonne qui partage un trait articulatoire est une structure branchante, ce qui garantirait la voyelle contre la réduction, qui se manifeste par un schwa. Le cas des voyelles qui ont en commun un trait articulatoire avec la consonne adjacente est en réalité le partage d'une primitive mélodique de type unaire (Anderson et Jones 1974, Kaye et al. 1985, Backley 2011). Nous verrons en section 4 que pour rendre compte des alternances des voyelles moyennes en coratin, nous nous baserons sur la théorie des éléments de Backley (2011) qui sera décrite en section 4.1. Il est également possible de formaliser cette alternance dans d'autres cadres théoriques. Quelques arguments en faveur de la TE seront exposés en section 4.1. Cependant, le but de cet article n'est pas de mettre en concurrence les théories entre elles.

Sous (4a) est représenté le partage de la primitive correspondant à la labialité (U) entre une consonne labiale $/ \mathrm{m} /$ et la voyelle labiale atone $/ \mathrm{o} /$ suivante. C'est ce partage de labialité qui permet à la structure de brancher. Pour cette raison, ce /o/ ne sera donc pas réduit puisqu'il est associé à deux points squelettaux, tandis que sous (4b), la voyelle palatale $/ \mathrm{i} /$ ne partage rien avec le $/ \mathrm{m} /$ précédent et c'est uniquement pour cette raison qu'elle est réduite à [ə] puisqu'elle n'est associée qu'à un seul point squelettal. 
(4) Maintien / réduction (structure branchante ou non)

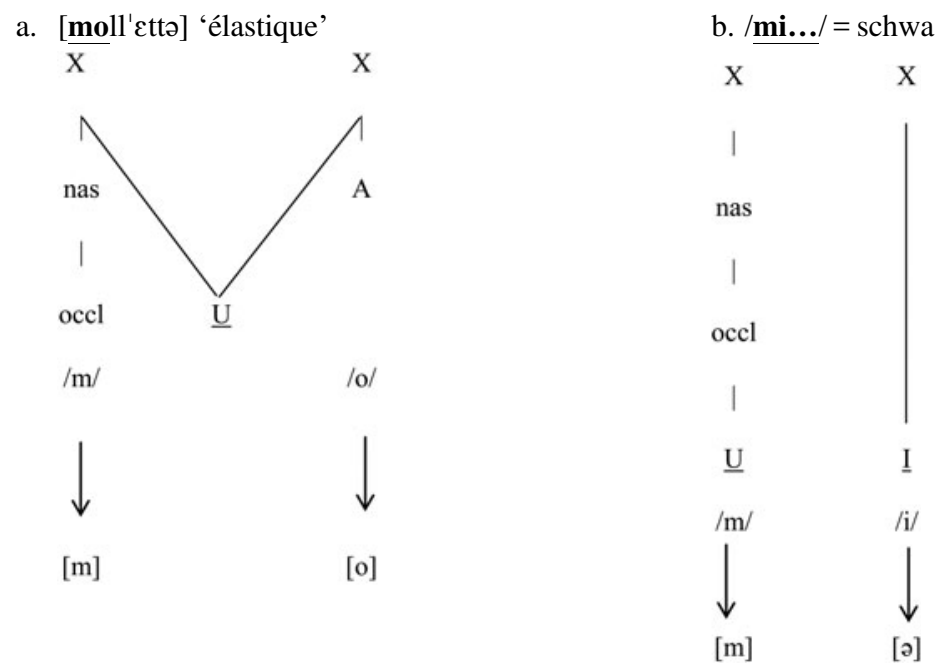

Examinons à présent les voyelles toniques, qui sont toujours réalisées pleines. Nous venons de voir que les voyelles qui branchent sont protégées contre la réduction. Partant du principe que s'il n'y a qu'une seule cause pour la nonréduction, on doit conclure que les voyelles toniques seraient aussi des structures branchantes, c'est-à-dire associées à deux points squelettaux. Par conséquent, la seule manière d'avoir une voyelle qui branche est de la considérer comme une voyelle structurellement longue.

\subsection{Voyelles toniques}

Cette partie montrera comment fonctionne l'allongement vocalique en position tonique pour l'italien ainsi que pour les dialectes méridionaux. Il sera présenté sous 3.2.3 le cas du coratin qui diffère des autres dialectes méridionaux.

\subsubsection{L'allongement tonique en italien}

L'allongement tonique est un phénomène bien connu et très étudié en italien (p.ex. Fava et Magno Caldognetto 1976, Bertinetto 1981) et dans d'autres langues comme en islandais (p.ex. Gussmann 2002). En effet, l'accent peut allonger les voyelles, comme en italien où le phénomène est bien attesté (Fava et Magno Caldognetto 1976, Bertinetto 1981, D'Imperio et Rosenthall 1999). Nous devons à Vogel (1982) la première analyse de l'allongement tonique avec en exemple l'italien où l'accent est matérialisé en tant qu'espace syllabique, c'est-à-dire une position squelettale (une position $\mathrm{X}$ ).

En ce qui concerne la représentation de l'accent, nous suivons Chierchia (1986), Larsen (1998) et Ségéral et Scheer (2008) qui proposent qu'il se matérialise en tant qu'espace syllabique, c'est-à-dire une position $[\mathrm{X}]$ vide impliquant ainsi que les voyelles toniques peuvent brancher sur cet espace comme présenté sous (5). 
En italien, les voyelles toniques sont longues en syllabe ouverte (5a) et brèves en syllabe fermée $(5 b)$. Les voyelles atones sont toujours brèves $(5 c)$.

(5) Accent tonique en italien

\begin{tabular}{|c|c|c|}
\hline $\begin{array}{l}\text { a. } \\
\text { [f'aato] } \\
\text { [p'iigro] }\end{array}$ & $\begin{array}{l}\text { 'destin' } \\
\text { 'fainéant' }\end{array}$ & $\begin{array}{l}*\left[\mathrm{f}^{\prime} \text { ato }\right] \\
*\left[\mathrm{p}^{\prime} \text { igro }\right]\end{array}$ \\
\hline $\begin{array}{ll}\text { b. } & {\left[f^{\prime} \text { atto }\right]} \\
& {\left[p^{\prime} \text { 'arko] }\right.}\end{array}$ & $\begin{array}{l}\text { 'fait' } \\
\text { 'parc' }\end{array}$ & $\begin{array}{l}*\left[\mathrm{f}^{\prime} \text { aatto }\right] \\
*\left[p^{\prime} \text { aarko }\right]\end{array}$ \\
\hline [ [bat $\widehat{\int}$ iino] & 'bassine' & \\
\hline
\end{tabular}

Dans les variétés d'italien décrites, la longueur vocalique n'est pas distinctive : elle n'est basée que sur des mesures phonétiques de durée. On peut donc se poser la question de savoir si ce type de phénomène phonétique doit être représenté en phonologie; Vogel (1982) et Chierchia (1986), par exemple, répondent par l'affirmative.

Dans le cadre auto-segmental, la quantité (des consonnes ou des voyelles) est encodée par le nombre de points squelettaux. Les consonnes et les voyelles sont longues si elles occupent deux positions, elles sont brèves si elles n'occupent qu'une seule position.

(6) Segment long vs court

a. court b. long

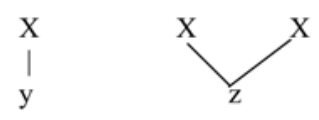

Si donc l'accent tonique allonge les voyelles et que cet allongement doit être représenté en phonologie, alors l'identité de l'accent tonique est de l'espace syllabique. C'est ce que concluent Vogel (1982) et Chierchia (1986) pour l'italien, et plus tard Larsen (1998), et Ségéral et Scheer (2008), plus généralement.

L'analyse de Chierchia (1986) sous (7) explique la restriction de l'allongement tonique aux syllabes ouvertes par une contrainte demandant que les rimes de la langue soient lourdes, c'est-à-dire dominent deux points squelettaux.

(7) Allongement tonique en italien

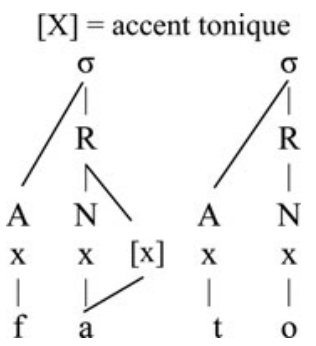

Sous (7), l'espace syllabique accentuel inséré permet de satisfaire la contrainte de rime lourde en italien. La voyelle tonique se réalise comme longue avec l'apport de l'espace syllabique de l'accent et permet de faire brancher la voyelle, satisfaisant ainsi 
la contrainte puisque le segment vocalique est en syllabe ouverte. Si la voyelle tonique était en syllabe fermée, l'espace accentuel ne pourrait pas être inséré puisque la rime serait déjà lourde. Par conséquent, la voyelle tonique sera brève.

\subsubsection{Le cas des dialectes méridionaux}

Il est bien connu dans la littérature que les dialectes parlés dans le sud-est de l'Italie (des Abruzzes aux Pouilles, et dans l'est de la Lucanie) ont des contraintes métricosyllabiques concernant certains processus phonologiques comme l'allongement vocalique qui peut se traduire en diphtongue, en contexte tonique (Rohlfs 19661969; Savoia 1987, 1990; Loporcaro 1988, 2001). Sous (8) sont présentées les différentes structures prosodiques pertinentes pour comprendre les faits.

(8) Structures prosodiques
a. C'V.CV\#
paroxyton en syllabe ouverte
b. C'VC.CVC\#
paroxyton en syllabe fermée
c. C'V.CV.CV\#
proparoxyton en syllabe ouverte
d. CV.CV.C'V\#
oxyton en syllabe ouverte

En effet, dans cette aire, ces processus sont conditionnés par la structure métricosyllabique du mot. Il y a une opposition entre le contexte (8a) où l'allongement vocalique en position tonique se manifeste comme une diphtongue et les contextes (8b) et (8c) où le processus est bloqué, c'est-à-dire que la voyelle reste brève. On peut comprendre facilement la raison pour laquelle en contexte (8b) la voyelle ne peut pas s'allonger : elle est entravée, ce qui implique que la rime est déjà lourde (cf. Chierchia 1986 et section 3.2.1). En revanche, on ne comprend pas du premier coup d'œil pourquoi sous (8c) et (8d) l'allongement vocalique n'opère pas, alors que la rime est légère. Il semblerait que dans ces contextes, l'effet est le même qu'en syllabe fermée, alors que la syllabe est ouverte. Loporcaro (2001 : 262) explique en termes de mores pourquoi la diphtongaison ne fonctionne pas dans ces contextes. Savoia (1987) fait de même pour d'autres dialectes. En effet, ce dernier propose que le contexte (8a) est bimoraïque, tandis que ( $8 b-d)$ sont monomoraïques, ce qui implique que la diphtongaison ne peut pas fonctionner (cf. 9 pour les dialectes de Tufillo et Palmoli dans les Abruzzes).

(9) Structure moraïque (Savoia 1987)

\begin{tabular}{|c|c|c|c|}
\hline more & more & more & \\
\hline$\left[\mathrm{k}^{\prime} \mathrm{e}\right.$ & $\mathrm{u}$ & fə] & 'je couds' \\
\hline$\left[\mathrm{k}^{\prime} \mathrm{e}\right.$ & $\mathrm{n}$ & fə] & 'tu comptes' \\
\hline$\left[\mathrm{k}^{\prime} \mathrm{e}\right.$ & $\int \partial$ & lə] & 'couds-le' \\
\hline [ve] & \multicolumn{2}{|c|}{.................. } & 'vous' \\
\hline
\end{tabular}

\begin{tabular}{|c|c|c|c|c|}
\hline \multicolumn{4}{|c|}{ Palmoli } & \multirow[b]{3}{*}{ 'doigt' } \\
\hline & more & more & more & \\
\hline e. & {$\left[\mathrm{d}^{\prime} \varepsilon\right.$} & $\mathrm{i}$ & tə] & \\
\hline f. & {$\left[\mathrm{d}^{\prime} \varepsilon\right.$} & to & rə] & 'doigts' \\
\hline g. & {$\left[\mathrm{t}^{\prime} \varepsilon\right.$} & $\mathrm{n}$ & drə] & 'cendre' \\
\hline 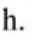 & {$[\operatorname{tr} \varepsilon]$} & \multicolumn{2}{|c|}{................ } & 'trois' \\
\hline
\end{tabular}


Sous (9a) et (9e), les voyelles toniques sont en contexte (8a) et par conséquent, l'allongement vocalique a lieu puisque, d'après l'analyse de Savoia (1987), il y a deux mores. À l'inverse, dans les données sous $(9 \mathrm{~b}-\mathrm{d})$ et $(9 \mathrm{f}-\mathrm{h})$, la voyelle tonique ne s'allonge pas, du fait qu'il n'y a qu'une more. C'est donc la structure métrique qui régit l'allongement de la voyelle dans ce type de dialecte.

Néanmoins, les données relevées sur le terrain montrent qu'en coratin, il y a bien des diphtongues en syllabe fermée (voir (10a) et (10b) pour les proparoxytons et (10c) pour les oxytons), à l'inverse des derniers travaux phonétiques publiés dans Romano (2012a, 2012b). D'après Melillo (1955 : 54), on trouve également à Ruvo di Puglia ${ }^{8}$ un mot comme [gal'iettə] 'galet' où la diphtongue se trouve en syllabe fermée; c'est le cas aussi d'après Savoia (2015 : 89), pour la même localité, avec

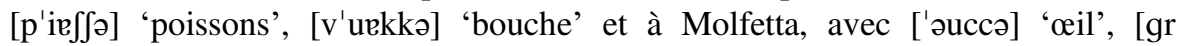
'oussə] 'gros' (Savoia 2015 : 91).

(10) Diphtongues en coratin

a. CVC

$\begin{array}{ll}\text { [ann'iəddə] } & \text { 'anneau' } \\ \text { [d'iəndə] } & \text { 'dent' } \\ \text { [f'uərt]ə] } & \text { 'ciseaux' } \\ \text { [m'uərtə] } & \text { 'mort' } \\ \text { [st'uəttsə] } & \text { 'morceau' } \\ \text { [gw'Errə] } & \text { 'guerre' } \\ \text { [-'iəddə] } & \text { 'suffixe DIM' }\end{array}$

b. Proparoxytons ${ }^{9}$

[ff'uommərə] 'pelote'

[g'uovətə] 'coude' c. Oxytons

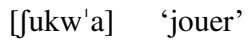

Il semblerait que les contraintes métrico-syllabiques vues supra ne fonctionnent pas pour le coratin. Dans la partie suivante, qui traitera de l'allongement tonique dans ce dialecte, nous verrons que l'analyse proposée est différente de l'approche traditionnelle relatée dans la littérature (Savoia 1987, 1990; Loporcaro 1988, 2001; Calabrese 2000; Romano 2012a, 2012b).

\subsubsection{L'allongement tonique en coratin}

Si l'on part du principe que les voyelles toniques sont longues en coratin de même qu'en italien, il y a deux désaccords considérables :

1. en coratin, toutes les voyelles toniques sont longues, y compris celles qui se trouvent en syllabe fermée;

2. le corrélat phonétique de la longueur n'est pas la durée comme en italien, mais la nonréduction.

Le premier point est illustré ci-dessous.

\footnotetext{
${ }^{8}$ Cette localité est située à environ $8 \mathrm{~km}$ de Corato.

${ }^{9}$ Notre corpus comporte peu de données pour les proparoxytons et les oxytons.
} 
(11) Voyelles en coratin

a. voyelle tonique en syllabe fermée

$\begin{array}{ll}\text { m'єfə } & \text { 'mieux' } \\ \text { kuv'єrtə } & \text { 'couverture' } \\ \text { r'ondənə } & \text { 'hirondelle' } \\ \text { t'urdə } & \text { 'hirondelle' } \\ \text { p'ombə } & \text { 'pompe' }\end{array}$

b. voyelle tonique en syllabe ouverte

$\begin{array}{ll}\text { l'imə } & \text { 'lime' } \\ \text { n'omə 'nom' } \\ \text { p'etə 'caillou' } \\ \text { m'utə 'entonnoir' } \\ \text { f'edə 'alliance' }\end{array}$

On peut rappeler que la durée (et donc la longueur) des voyelles n'était déjà pas conditionnée par la structure syllabique en latin classique, où l'on trouvait donc des voyelles longues en syllabe fermée. Une telle indépendance de la durée par rapport à la structure syllabique a cependant disparu avec la « révolution prosodique » (Loporcaro 2011 : 53) qui a mené à l'évolution du latin classique vers le roman, la distinction de longueur vocalique ayant laissé la place à une distinction de timbre. En effet, le système à dix voyelles (longues ou brèves) du latin classique est passé au système proto-roman /i, e, $\varepsilon$, i, o, o, u/ (Bourciez 1930, Loporcaro 2011).

Le traitement traditionnel du processus affectant la longueur des voyelles toniques latines a été étudié en phonologie diachronique des langues romanes comme étant essentiellement conditionné par la structure syllabique, c'est-à-dire selon l'ouverture ou la fermeture de la syllabe (CV ou CVC). La structure de la syllabe est en relation directe avec certains processus impliquant des changements de quantité du noyau syllabique (allongement, brièveté). L'allongement tonique peut être réalisé comme une diphtongue indépendamment de la quantité syllabique.

On peut classer les langues romanes sur des bases de changements des voyelles toniques conditionnées par la structure syllabique en deux grands groupes :

- celles qui n'ont pas de différence de traitement en syllabe ouverte ou en syllabe fermée, comme le dialecte de Pozzuoli (Russo 2010), mais aussi le coratin;

- celles qui ont une différence de traitement d'après le conditionnement syllabique, comme le français et l'italien.

La longueur vocalique peut se manifester en surface soit comme de la longueur phonétique, ce qui est le cas en français, en italien et dans d'autres langues, soit par un autre objet en surface comme en coratin, c'est-à-dire par la (non-)réduction (cf. longueur virtuelle infra).

Les deux représentants des deux types de langues sont donc le coratin pour le type A et l'italien pour le type B. Le tableau sous (12) présente la situation de la longueur vocalique dans les deux types de langues. Dans le type A, les voyelles toniques peuvent être longues en syllabe fermée, alors que ce n'est pas le cas pour le type B.

(12) Tableau de la longueur vocalique en position tonique

\begin{tabular}{|l|c|c|}
\hline & A & B \\
\hline CV & VV & VV \\
\hline CVC & VV & V \\
\hline
\end{tabular}


En coratin et dans d'autres dialectes italiens, la manifestation en surface de cette longueur vocalique peut se manifester avec des diphtongaisons descendantes qui sont appelées frangimenti se définissant par un type d'altération du timbre vocalique accentué (cf. Giammarco 1973; Loporcaro 1988; Savoia 1990, 2015; Avolio 1995; Grassi, Sobrero et Telmon 2003; Abete 2005, 2006; Savoia et Carpitelli 2008; Romano 2012a, 2012b). Ce processus de diphtongaison peut se réaliser en syllabe ouverte, mais aussi en syllabe fermée en coratin, comme nous l'avons déjà vu en section 3.2.3 et le verrons ci-dessous.

\section{(13) Frangimento en coratin}

a. syllabe ouverte : b. syllabe fermée :

$\begin{array}{llll}\text { [c'ainə] } & \text { 'chien' } & \text { [l'oup] } & \text { 'loup' } \\ \text { [p'iedə] } & \text { 'pied' } & \text { [fr'ceddə] } & \text { 'froid' } \\ \text { [bb'ounə] } & \text { 'bon' } & \text { [d'iəndə] } & \text { 'dent' }\end{array}$

Les conséquences de la longueur en surface peuvent être réalisées de différentes manières, comme il a été dit précédemment, en coratin par la non-réduction, la longueur phonétique ou le frangimento. Le tableau sous (14) récapitule ces processus pour les langues de type A et B. La prédiction posée est la suivante : si le coratin cumule à la fois la non-réduction et le frangimento alors tous les dialectes de type A ne peuvent pas faire autrement et auront donc les mêmes caractéristiques que le coratin. En revanche, dans les dialectes de types B, la longueur phonétique brute, que ce soit de la longueur ou du frangimento, est variable puisqu'elle est conditionnée par la structure syllabique sans qu'il y ait de réduction vocalique.

(14) Tableau des conséquences de la longueur

\begin{tabular}{|l|l|l|l|l|}
\hline manifestations de surface & \multicolumn{2}{|c|}{ A } & \multicolumn{2}{c|}{ B } \\
\hline & $\mathrm{CV}$ & $\mathrm{CVC}$ & $\mathrm{CV}$ & $\mathrm{CVC}$ \\
\hline non-réduction & + & + & - & - \\
\hline frangimento & $\mathrm{VV}$ & $\mathrm{VV}$ & $\mathrm{VV}$ & $\mathrm{V}$ \\
\hline longueur phonétique & $\mathrm{VV}$ & $\mathrm{VV}$ & $\mathrm{VV}$ & $\mathrm{V}$ \\
\hline
\end{tabular}

Une fois établie la différence entre le coratin et l'italien, nous pouvons observer que sous (15a), l'espace accentuel permet à la voyelle de brancher et par conséquent d'être maintenue. En revanche, sous (15b), la voyelle demeure réduite puisqu'elle est réalisée brève en l'absence d'unité $[\mathrm{X}]$ apportée par l'accent. Il est important de signaler que pour cette analyse, le schwa n'est que de surface : lorsque le /u/ sous (15b) est atone, il s'agit toujours d'un $u$ dans la représentation phonologique, puisque son identité mélodique n'a pas été modifiée par la computation phonologique. Il acquiert son identité de voyelle pleine ou de voyelle réduite (schwa) uniquement durant l'interprétation phonétique de la structure phonologique (Gussmann 2007, Scheer 2010, Cyran 2012). 
(15) Représentations des voyelles toniques et atones

a. Voyelle tonique b. Voyelle atone

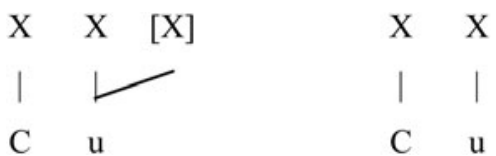

Cette perspective dite de « longueur virtuelle ${ }^{10}$ suppose qu'il y ait une différence entre les représentations phonologiques d'un objet et leur réalisation phonétique (cf. Lowenstamm 1991). Cette longueur virtuelle peut toucher aussi bien les voyelles que les consonnes. Le coratin n'a pas d'opposition de longueur vocalique en surface et remplit donc les conditions nécessaires. Dans ce système, les voyelles sous-jacemment longues, c'est-à-dire qui branchent, sont réalisées pleines, tandis que les voyelles sous-jacemment brèves sont réalisées schwa : voir (16).

(16) Longueur virtuelle en coratin

$\begin{array}{ll}/ \mathrm{vv} / & / \mathrm{v} / \\ {[\mathrm{i}, \mathrm{e}, \varepsilon, \mathrm{o}, \mathrm{o}, \mathrm{u}, \mathrm{a}]} & {[\mathrm{\partial}]} \\ {[\mathrm{u}]} & {[\vartheta]}\end{array}$

À présent, nous avons tous les éléments nécessaires pour exposer les données et analyser le processus d'alternance des voyelles moyennes où il faudra néanmoins introduire la Théorie des éléments proposée par Backley (2011).

\section{L'ALTERNANCE DES VOYELLES MOYENNES EN CORATIN}

Après avoir passé en revue la réduction/le maintien des voyelles en coratin, nous pouvons à présent analyser le processus d'alternance des voyelles moyennes dans ce dialecte.

Nous avons constaté précédemment que les voyelles atones ne sont pas réduites lorsqu'elles sont adjacentes à une consonne qui partage un trait articulatoire. Les données sous (17) et (18) montrent l'alternance des voyelles moyennes où notamment l'ATRité de la voyelle tonique change et où, dans un contexte bien particulier, la hauteur relève.

(17) Alternance des voyelles moyennes

\begin{tabular}{|c|c|c|c|}
\hline p'ontə & 'pointe' & pondar' $\varepsilon$ ddə & 'pointe-DIM' \\
\hline mb' $\underline{\partial}$ ddə & 'gonfleur' & mboddət $\int \varepsilon$ ddə & 'gonfleur-DIM' \\
\hline m'วllə & 'élastique' & moll'cttə & 'élastique-DIM' \\
\hline p'ōmbo & 'pompe' & pombar' $\varepsilon$ ddə & 'pompe-DIM' \\
\hline $\mathrm{r}^{\prime} \underline{\varepsilon} \mathrm{c} c \boldsymbol{\partial}$ & 'oreilles' & reccot' $\varepsilon$ ddə & 'oreilles-DIM' \\
\hline $\mathrm{n}^{\bar{\varepsilon}} \boldsymbol{\varepsilon}_{\mathrm{U}}$ & 'brouillard' & neff'usə & 'qui est envahi par brouillard' \\
\hline$\widehat{\mathrm{d} z^{\prime} \varepsilon} \varepsilon$ nค & 'idée' & dzenn'onə & ‘idée-AUGM’ \\
\hline $\mathrm{c}^{\prime} \varepsilon \overrightarrow{\mathrm{k}} \partial$ & 'pli' & cek'a & 'plier' \\
\hline
\end{tabular}

${ }^{10}$ Le concept de longueur virtuelle ne peut être viable que dans un système qui ne possède pas d'opposition de longueur en surface et qui peut distinguer des longues et des brèves en phonologie. 


\begin{tabular}{|c|c|c|c|}
\hline k'ondzə & 'teinture' & kundzat'orə & 'tanneur' \\
\hline k'ōrnə & 'corne' & kürnətf'iəddə & 'corne-DIM' \\
\hline nəg'? & 'magasin' & nəgüddz'jandə & 'commerçant' \\
\hline k'ordə & 'corde' & kurd'cttə & 'corde-DIM' \\
\hline
\end{tabular}

(18) Récapitulatif des alternances des voyelles en position atone
a. [0] tonique
$\rightarrow \quad[\mathrm{o}]$ atone $/ \mathrm{C}$ lab
[p'óvərə]
[povər'iəddə]
b. $[\varepsilon]$ tonique
$\rightarrow \quad[\mathrm{e}]$ atone $/ \mathrm{C}$ pal $\quad\left[\mathrm{n}^{\prime} \boldsymbol{\varepsilon}\right.$ fə]
[neff'use]
c. [ग], [o] tonique
[u] atone $/ \mathrm{C}$ vél
[k'ondzə]
[kundzat'oro]

Examinons les exemples sous $(18 \mathrm{a}-\mathrm{b})$ où nous pouvons observer que l'ATRité des voyelles moyennes alterne dans la mesure où les voyelles moyennes mi-ouvertes $/ \varepsilon, o /$ se réalisent mi-fermées [e,o] en position non-tonique. En revanche, sous (18c), nous ne sommes pas dans un cas d'alternance d'ATRité mais dans un contexte de hauteur, puisque les voyelles $/ \mathrm{o}, \mathrm{J} /$ font surface en position atone par un $[\mathrm{u}]$ uniquement lorsqu'elles sont adjacentes à une consonne vélaire. Les alternances sous (18a) et (18b) n'ont absolument rien d'étonnant puisqu'elles sont aussi attestées en italien standard (Krämer 2009 : 100). Krämer montre sous (19) que les voyelles -ATR ne peuvent pas faire surface en position atone puisqu'elles sont réalisées dans leur version + ATR.

(19) Alternance en italien standard des voyelles moyennes

$$
\begin{aligned}
& \text { [ortop' } \varepsilon \text { diko] 'orthopédiste' [ortoped'ia] 'orthopédique' } \\
& \text { [l'odzika] 'logique' [lodz̧ikam'ente] 'logiquement' }
\end{aligned}
$$

Comme nous l'avons précisé précédemment, la description de la structure interne des segments proposée par Backley (2011) dans sa théorie des éléments permettra de comprendre l'alternance des voyelles moyennes en coratin.

\subsection{La théorie des éléments de Backley (2011)}

La structure interne des segments est composée de primitives mélodiques de type unaire dont la composition peut produire des consonnes ou des voyelles. En outre, on trouve aussi des primitives constituées de traits distinctifs de type binaire. En revanche, les primitives de types unaires sont plus grandes que les traits distinctifs puisqu'il faut plusieurs traits pour décrire le même objet qu'une primitive unaire. De plus, une caractéristique importante propre à une primitive unaire (contrairement à un trait) est que la première a une prononciation, tandis qu'un trait n'en a pas. Cela revient à dire que chaque primitive unaire est indépendamment prononçable. Le choix de la TE plutôt que d'une autre théorie découle des faits suivants :

- la binarité $(+\mathrm{ou}-)$ force la grammaire à produire des prédictions incorrectes puisque la grammaire se sert de propriétés de valeurs négatives phonologiquement inactives. Ce procédé est redondant et surgénère dans la mesure où la bivalence prédit la possibilité de nombreux processus phonologiques qui n'existent pas, tandis que l'approche unaire utilise des traits monovalents permettant d'identifier un trait seulement si celui-ci est positif. Cette perspective restreint la grammaire puisqu'elle n'implique que les traits participants activement à la phonologie; 
- l'autre argument de poids en faveur de la TE est qu'elle capture la connaissance linguistique partagée par le locuteur et l'auditeur par le biais d'éléments. Ces derniers sont des caractéristiques acoustiques du son articulé. Le but de la TE est de ne favoriser ni l'auditeur ni le locuteur, mais les connaissances linguistiques qu'ils partagent. Ce partage opère à travers l'intermédiaire du son articulé qui agit entre l'origine du bruit (organes vocaux) et sa cible (l'appareil auditif de l'auditeur).

La description de la structure interne des segments que l'on trouve dans la théorie des éléments de Backley (2011) qui est, à ma connaissance, la version la plus récente découlant de la phonologie du gouvernement (Kaye et al. 1985, Harris et Lindsey 1995), comporte six éléments :

- éléments vocaliques : |I|, |U|, |A|

- éléments consonantiques : $|\mathrm{H}|,|\mathrm{L}|,|\mathrm{P}|$

Comme il a été précisé supra, les éléments sont individuellement interprétables, c'est-à-dire que l'élément $|\mathrm{I}|$ est la réalisation de la voyelle [i], l'élément $|\mathrm{A}|$, la réalisation de la voyelle [a], etc. Nous verrons plus loin dans l'article que les éléments consonantiques et vocaliques peuvent se combiner pour former des segments plus complexes.

\subsubsection{Les éléments vocaliques et la notion de tête}

Dans le cadre de la TE, les éléments vocaliques sont constitués des trois voyelles cardinales du triangle vocalique qui se trouvent, associées, dans toutes les langues du monde : $|\mathrm{I}|,|\mathrm{U}|,|\mathrm{A}|$. Chaque voyelle est constituée d'un élément ou de la fusion de deux ou plusieurs éléments, comme on peut le voir sous (20).

(20) Éléments vocaliques simples et combinés

a. éléments simples b. éléments combinés

$$
\begin{aligned}
& |\mathrm{I}|=[\mathrm{i}] \quad|\mathrm{AI}|=[\mathrm{e}] \quad|\mathrm{IAU}|=\varnothing \\
& |\mathrm{U}|=[\mathrm{u}] \quad|\mathrm{AU}|=[\mathrm{o}] \\
& |\mathrm{A}|=[\mathrm{a}] \quad|\mathrm{IU}|=[\mathrm{y}]
\end{aligned}
$$

Traditionnellement en TE, les voyelles centrales que l'on peut qualifier de schwa (moyen, haut, bas) sont analysées en tant qu'absence de primitives mélodiques. Cependant, l'identité de ces schwa peut être une voyelle froide $\left(\mathrm{v}^{\circ}\right)$ chez Kaye et al. (1985) ou encore l'élément neutre @ chez Harris (1994). Backley leur assigne l'identité $|\mathrm{A}|$, par exemple en anglais.

La TE utilise la notion de tête pour exprimer une asymétrie hiérarchique. Cette relation asymétrique s'exprime en TE par la notion de tête, renvoyant à la différence entre un élément dit proéminent (la tête) et les autres éléments (opérateurs). Dans un système à sept voyelles comme celui du coratin (et un schwa quand la réduction opère), la relation de tête vs non-tête est représentée sous (21).

(21) Système vocalique du coratin (Bucci 2013)

i $|\underline{I}|$

e |IA|

$$
\text { o }|\underline{U A}|
$$

$\varepsilon|\mathrm{IA}|$

๑ |UA|

a $\underline{\mid A} \mid$ 
Le paramètre de tête permet dans ce système de rendre compte du contraste des voyelles moyennes où $\mathrm{e} \sim \varepsilon$ et $\mathrm{o} \sim \jmath$. En coratin, les voyelles moyennes sont distinguées par la présence d'une tête pour les versions + ATR, et son absence pour les variantes -ATR. Nous rappelons que la voyelle schwa, qui se manifeste quand la réduction opère, est en réalité une voyelle qui a ses éléments, mais qui ne branche pas, ce qui implique qu'elle est interprétée en surface comme un schwa et non pas comme une voyelle qui perd ses éléments.

Un paramètre propre à la TE de Backley (2011) est qu'elle différencie la palatalité et la labialité justement en utilisant la proéminence de l'élément $|\mathrm{U}|$ pour les labiales qui ont un $|\underline{U}|$, alors que pour la vélarité, $|U|$ est opérateur. Nous verrons plus tard, lors de l'analyse en section 5, que cette asymétrie permettra de rendre compte des alternances vues précédemment.

\subsubsection{Les éléments consonantiques}

Backley (2011) propose une description complète des segments consonantiques et c'est le seul ouvrage sur la TE, à ma connaissance, qui se propose de décrire chaque segment. ${ }^{11}$ En effet, dans Kaye et al. (1985), la structure interne des consonnes n'est pas abordée et dans Harris (1994) et Harris et Lindsey (1995), seulement quelques segments consonantiques sont décrits. Pour cette raison, cette version de la TE rend explicite, à notre sens, la structure interne des consonnes que l'on trouve dans les systèmes des variétés des dialectes des Pouilles. Les éléments consonantiques sont au nombre de trois et peuvent être combinés entre eux, mais aussi avec les éléments vocaliques.

L'élément $|\mathrm{H}|$ correspond aux hautes fréquences (High frequency) que l'on trouve dans les occlusives au moment de l'explosion lors du relâchement des articulateurs après l'occlusion, mais également dans les consonnes fricatives et lors de l'aspiration.

L'élément |?| renvoie à une occlusion ou à un coup de glotte que l'on trouve notamment dans les consonnes occlusives. En effet, il caractérise une tenue puis un relâchement brusque des articulateurs. On trouve aussi cet élément dans les consonnes ayant comme propriété une résonance glottale.

Et pour terminer, l'élément |L| se caractérise par la basse fréquence (Low frequency) où il cumule les fonctions de $|\mathrm{N}|$ et $|\mathrm{L}|$ dans la TE de Harris (1994). Il est associé à un abaissement du voile du palais, impliquant que cet élément se trouve dans les nasales. Il permet aussi de différencier les consonnes voisées des nonvoisées lorsqu'il est associé à l'élément $|\mathrm{H}|$.

Appliquons maintenant la description des segments consonantiques de Backley (2011) au système du coratin qui possède 22 segments consonantiques. Une partie de ces consonnes sont décrites sous (22). Celles notées en gras sont pertinentes pour l'analyse qui sera proposée infra.

${ }^{11}$ C'est la raison du choix de cette version de TE par rapport à une version antérieure. En effet, l'ouvrage est complet puisque toute les consonnes et les voyelles sont décrites dans plusieurs systèmes. 
(22) Inventaire segmental des consonnes du coratin ${ }^{12}$

$$
\begin{aligned}
& {[\mathbf{p}]=|\underline{\mathrm{U}} \boldsymbol{P}(\mathbf{H})|} \\
& {[\mathrm{b}]=\mid \underline{\mathrm{U}} \text { ? (H) } \underline{\mathrm{L}} \mid} \\
& \text { [f] }=|\underline{\mathrm{U}} \mathrm{A} \mathrm{H}| \\
& {[\mathrm{v}]=|\underline{\mathrm{U}} \mathrm{A} \mathrm{H} \mathrm{L}|} \\
& {[\mathbf{m}]=|\underline{\mathbf{U}} \boldsymbol{?}(\mathbf{H}) \mathbf{L}|} \\
& {[\mathrm{n}]=\mid \mathrm{A} \text { ? (H) L } \mid} \\
& {[\mathrm{t}]=|\mathrm{A} ?(\mathrm{H})|} \\
& \text { [d] = |A ? (H)L } \underline{\text { L }} \\
& {[\mathrm{k}]=\mid \mathrm{U} \text { ? (H)| }} \\
& {[\mathrm{g}]=\mid \mathrm{U} \text { ? (H) L } \underline{\mathrm{L}}} \\
& {[\mathrm{c}]=|\underline{\mathrm{I}} \mathbf{?}(\mathbf{H})|} \\
& {[\mathbf{J}]=\mid \underline{I} \mathbf{P}(\mathbf{H}) \underline{\mathbf{L}} \text { | }} \\
& {[\mathrm{C}]=|\underline{\mathrm{I}} \mathrm{H}|} \\
& \text { [3] }=|\underline{I} \mathrm{H} \mathrm{L}| \\
& {[\mathrm{s}]=|\mathrm{A} \mathrm{H}|}
\end{aligned}
$$

Nous avons vu en section 3.1 sous (4) qu'une structure peut brancher entre une voyelle atone et sa consonne adjacente uniquement s'il y a un partage d'éléments entre les deux segments, c'est-à-dire soit l'élément labial |Ü|, soit l'élément vélaire $|\mathrm{U}|$ ou encore l'élément palatal $|\underline{\mathrm{I}}|$. C'est ce partage, et uniquement ce partage, qui permet à la voyelle de brancher et par conséquent d'être maintenue.

\section{Analyse}

L'analyse de la réduction/du maintien vocalique vue en section 3 a unifié les deux contextes de maintien des voyelles : une voyelle qui branche sur deux points squelettaux n'est pas réduite à schwa. Nous avons également vu que cette perspective suppose qu'il y a une différence entre les représentations phonologiques et les réalisations phonétiques (longueur virtuelle).

L'hypothèse faite dans cette analyse repose sur le fait que le partage d'une primitive mélodique (ici un élément) serait en réalité l'éviction de la primitive appartenant à la voyelle par la primitive de la consonne. Ainsi, dans ce type de structure où une primitive appartenant à une consonne est également interprétée par un noyau, ce dernier ne peut interpréter un autre élément que si l'élément en commun avec l'attaque est proéminent, c'est-à-dire tête. Cette hypothèse est illustrée sous (23) où l'on peut observer sous (23a) les structures bien formées, alors que sous (23b), la structure est mal formée.

(23) Partage d'éléments

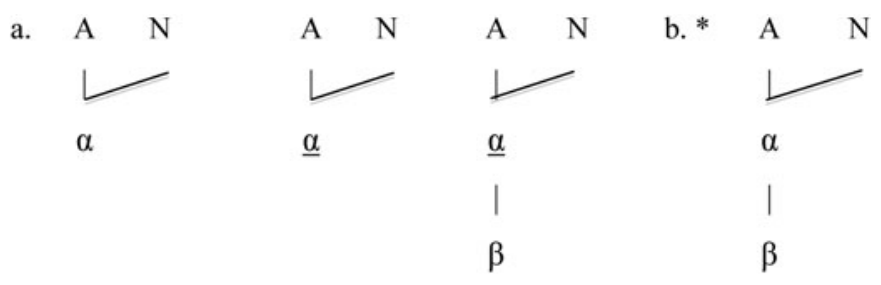

Nous montrerons que ce principe permettra de rendre compte des alternances des voyelles moyennes en coratin, récapitulées sous (24).

\footnotetext{
${ }^{12}$ Certains segments comportent deux têtes dans leurs structures internes afin de distinguer les nasales $[\mathrm{n}]$ et $[\mathrm{m}]$ des occlusives voisées [d] et [m]. En effet, l'élément $|\mathrm{L}|$ est contenu dans les consonnes voisées et nasales.
} 
(24) Alternances des voyelles moyennes en coratin

a. $\rho \sim \mathrm{o} / \mathrm{lab}, \varepsilon \sim \mathrm{e} / \mathrm{pal}$ (ATRité)

b. $\mathrm{o}, \mathrm{o} \sim \mathrm{u}$ (hauteur)

L'idée que seuls les éléments tête peuvent être partagés repose sur l'asymétrie proposée par Backley (2011) concernant les segments labiaux et vélaires où U peut être tête ou non. Cette perspective est bien connue en phonologie du gouvernement en tant que Licensing Contraints (contraintes de licenciement); voir Charette et Göksel 1994, 1996; Kaye 2001; Scheer 2010). Il faut tout de même préciser que les contraintes de licenciement ont été développées pour expliquer les restrictions des propriétés combinatoires entre éléments. A priori, aucune langue ne produit toute la gamme de segments possibles engendrés des combinaisons réalisables des éléments. Ces contraintes permettent ainsi de définir le choix particulier qu'une langue fait des possibilités combinatoires, mais elles participent aussi à définir les propriétés des processus phonologiques de la langue. En ce qui concerne le coratin, les contraintes de licenciement sont les suivantes :

(25) Contraintes de licenciement

a. lorsqu'elles le peuvent, les voyelles partagent les primitives définissant le lieu d'articulation avec les consonnes adjacentes;

b. la consonne impose sa mélodie à la voyelle adjacente et si son élément est tête, elle impose sa tête à la voyelle;

c. contrainte de licenciement A : un noyau qui partage une primitive mélodique avec une consonne adjacente ne peut licencier un opérateur que si l'élément partagé est tête (voir 23);

d. contrainte de licenciement B : l'élément $|\mathrm{A}|$ ne peut être partagé entre une voyelle et une consonne.

\subsection{Cas basique de l'alternance et du maintien vocalique : alternance $\varepsilon \sim \mathrm{e}$}

Il est maintenant temps d'éprouver les contraintes de licenciement vues sous (23) et (25) en examinant l'alternance des voyelles $/ \varepsilon, \mathrm{o} /$ qui se réalisent en position atone [e] et [o] lorsqu'elles sont adjacentes à une consonne qui partage une primitive mélodique. Sous (26a), la voyelle $\varepsilon$ est composée de $|\mathrm{I}|$ et de $|\mathrm{A}|$ et aucun des éléments ne possède une tête. Elle est maintenue puisqu'elle branche sur l'espace apporté par l'accent.

(26) a. [c'ckə] 'pli'

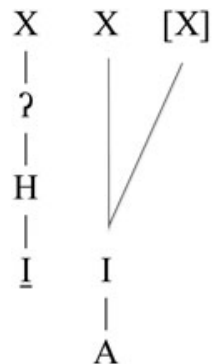

$[\mathrm{c} \quad ' \varepsilon$

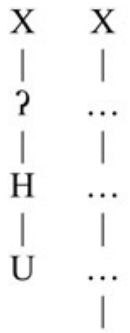

$\mathrm{k} \quad$ ə] b. [cek'a] 'plier'

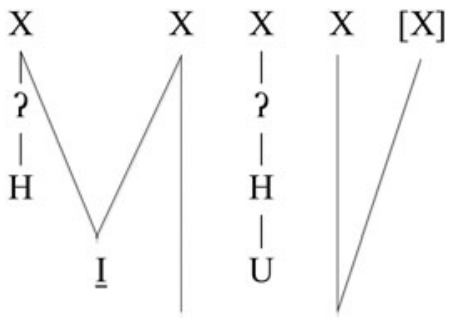

A

$\underline{\mathrm{A}}$

$\left[\begin{array}{llll}\mathrm{c} & \mathrm{e} & \mathrm{k} & \text { 'a }\end{array}\right]$ 
Nous savons maintenant que le système de Backley (2011) mentionne que les consonnes palatales contiennent un |I| tête. Sous (26b), la voyelle du radical n'est plus sous l'accent mais elle échappe à la réduction puisqu'elle est adjacente à une consonne palatale $c$. Cependant, on observe que celle-ci fait surface sous sa forme +ATR [e] lorsqu'elle est atone et maintenue. Ce qui différencie (26a) de (26b) est la nature du maintien. En effet, dans le premier cas, le branchement se réalise sur l'unité $[\mathrm{X}]$ accentuelle tandis que dans le second, il opère sur la consonne palatale. Par conséquent, c'est cette différence de nature du branchement qui doit être responsable de l'alternance $\varepsilon \sim$ e.

D'après les contraintes de licenciement et l'identité segmentale des segments vocaliques du coratin vues supra, on déduit que la voyelle du radical, lexicalement -ATR, est sans tête mais acquiert une tête $|\underline{I}|$ puisque la consonne palatale branche sur elle. En effet, la consonne palatale $c$ a dans sa structure interne un $|\underline{I}|$ qui est imposé au noyau atone adjacent en incluant un opérateur $|\mathrm{A}|$ puisque le $|\mathrm{I}|$ partagé avec la consonne voisine est tête. Ainsi, la structure satisfait la contrainte de licenciement (25c).

\subsection{Cas basique de l'alternance et du maintien vocalique : alternance $\supset \sim 0$}

Sous (27a), la voyelle $\supset$ est composée de U et A et aucun des éléments ne possède une tête. Elle est maintenue puisqu'elle branche sur l'espace apporté par l'accent.

(27) a

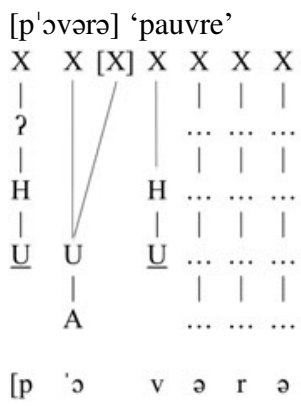

b. [povər'iddə] 'pauvre-DIM'

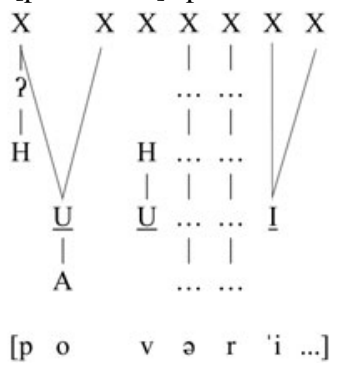

Backley (2011) mentionne que les consonnes labiales contiennent un |UI tête. Sous (27b), la voyelle du radical n'est plus sous l'accent, mais elle échappe à la réduction puisqu'elle est adjacente à une consonne labiale $p$. Cependant, on observe que celle-ci fait surface sous sa forme + ATR [o] lorsqu'elle est atone et maintenue. Ce qui différencie (27a) de (27b) est la nature du maintien. En effet, dans le premier cas, le branchement se réalise sur l'unité $[\mathrm{X}]$ accentuelle tandis que dans le second, il opère sur la consonne labiale. Par conséquent, c'est cette différence de nature du branchement qui doit être responsable de l'alternance $0 \sim 0$.

D'après les contraintes de licenciement et l'identité segmentale des segments vocaliques du coratin vues supra, on en déduit que la voyelle du radical, lexicalement -ATR et sans tête, acquiert une tête |Ü puisque la consonne labiale branche sur elle. En effet, la consonne labiale $p$ a dans sa structure interne un $|\underline{U}|$ qui est imposé au noyau atone adjacent en incluant un opérateur $|\mathrm{A}|$ puisque le $|\mathrm{U}|$ partagé avec la 
consonne voisine est tête. Ainsi, la structure satisfait donc la contrainte de licenciement (25c).

\subsection{Cas basique de l'alternance et du maintien vocalique : alternance $5 / 0 \sim u$ dans un contexte vélaire}

Le dernier cas d'alternance se manifeste uniquement dans un contexte de vélarité et c'est uniquement dans ce cas que l'on observe l'alternance vue sous (18c), c'est-àdire par exemple que $/ \mathrm{o} /$ adjacent à une consonne vélaire est maintenu en position atone; le résultat obtenu n'est pas le [o] attendu mais [u] : [k'ondzə] 'teinture'; [kundzat'orə] 'tanneur'. Il faut préciser deux faits importants :

- il n'y a pas d'équivalent pour les voyelles antérieures; /ع/ adjacent à une consonne palatale se réalise [e] (et non pas [i]) en position atone;

- Cela se produit avec les consonnes vélaires, mais pas avec les consonnes labiales; /o/ se réalise [o] (et non pas $[\mathrm{u}]$ ) en présence d'une consonne labiale.

Par conséquent, on en conclut que cette alternance est propre d'une part aux voyelles postérieures et d'autre part aux consonnes vélaires. Or la particularité de ces dernières dans le cadre de Backley est de posséder un élément |U| qui n'est pas tête, alors qu'il est tête dans les labiales. Ainsi, les particularités de ce type d'alternance doivent découler de ce fait.

La contrainte de licenciement proposée sous (25c) permet de solutionner et de rendre compte de ce type d'alternance. La représentation sous (28a) montre le branchement de la voyelle radicale sur l'espace accentuel. Tandis que sous (28b), la consonne vélaire impose son élément |U| à la voyelle et ce partage garantit cette dernière contre la réduction. Le $|\mathrm{U}|$ partagé ne peut tolérer la présence d'un $|\mathrm{A}|$ réalisé dans le noyau radical puisqu'il n'est pas tête : la voyelle radicale / / perd alors son élément | $\mathrm{A} \mid$, impliquant qu'elle se réalise [u] lorsqu'elle est en contact avec une consonne vélaire (cf. la contrainte 25c).

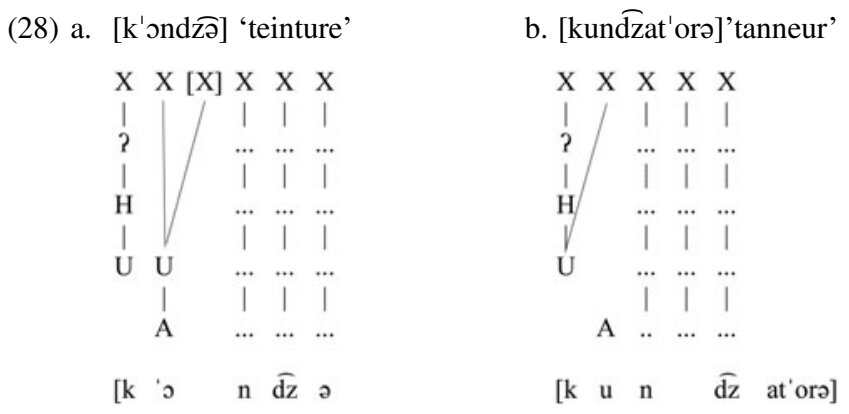

Les représentations sous (28) montrent l'alternance de la voyelle /o/ se réalisant [u] lorsqu'elle est atone et adjacente à une consonne vélaire (p.ex. [k'ordə] 'corde'; [kurd'cttə] 'corde-DIM'). Cette alternance fonctionne bien entendu avec la consonne vélaire voisée (p.ex. [vərg'onjə] 'honte'; [vergunn'usə] 'honteux').

Il s'agit du même principe vu supra, c'est-à-dire la contrainte de licenciement proposée sous (25c) stipulant qu'un noyau partageant un élément avec une consonne 
adjacente ne peut licencier un opérateur que si l'élément partagé est tête. La représentation sous (29a) montre le branchement de la voyelle radicale sur l'espace accentuel, tandis que sous (29b), la consonne vélaire impose son élément $|\mathrm{U}|$ à la voyelle (qui, elle, a un élément $|\underline{\mathrm{U}}|$ ) et ce partage garantit cette dernière contre la réduction. Le $|\mathrm{U}|$ imposé et partagé ne peut tolérer la présence d'un $|\mathrm{A}|$ réalisé dans le noyau radical puisqu'il n'est pas tête; alors la voyelle radicale /o/ perd son élément $|\mathrm{A}|$, impliquant qu'elle se réalise [u] lorsqu'elle est en contact avec une consonne vélaire.

(29) a. [k'ordə] 'corde'

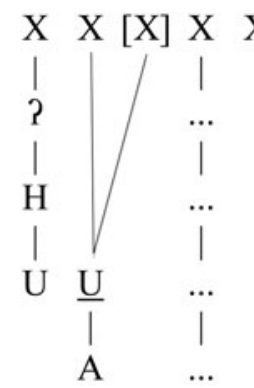

$\left[\begin{array}{lllll}\mathrm{k} & \mathrm{o} & \mathrm{r} & \mathrm{d} & \text { ə) }\end{array}\right]$ b. [kurd'ettə] 'corde-DIM'

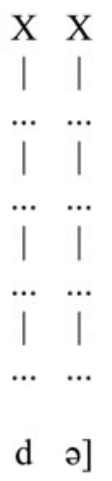

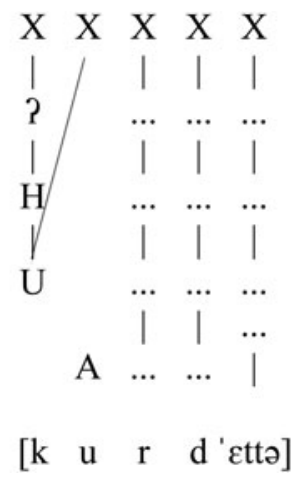

\subsection{Cas basique : la réduction}

Pour terminer l'analyse, considérons à présent le cas où la voyelle est réduite en position atone. Nous savons qu'une voyelle qui ne branche pas est réduite à schwa. Les conditions pour qu'une voyelle soit réduite sont qu'elle doit être en position atone et qu'elle ne partage pas de primitive mélodique avec la consonne adjacente. Sous (30a), la voyelle tonique $e$ ne réduit pas, puisqu'elle peut brancher sur l'espace syllabique apporté par l'accent, tandis que sous (30b) elle n'a pas cette possibilité, puisque l'accent est sur le suffixe. La voyelle $e$ est réduite à schwa dans la mesure où la consonne adjacente à sa gauche est dentale $(t)$ et celle à sa droite est labiale. Par conséquent, la voyelle ne branche pas.

Néanmoins, on observe que la voyelle antérieure contient un élément $|\mathrm{A}|$ et, qu'a priori, elle pourrait le partager avec la consonne dentale adjacente, ce qui la garantirait contre la réduction. Or, l'observation dans la langue est que les dentales sont toujours neutres quant à la réduction, c'est-à-dire qu'elles ne permettent à aucune voyelle d'échapper à la réduction. Il y a alors deux solutions : les dentales contiennent l'élément A comme le prévoit Backley (2011) (à la suite des travaux londonien réalisés à SOAS dans les années 90 ), et il faut alors retranscrire l'impossibilité de partage de l'élément $\mathrm{A}$ entre une consonne et une voyelle, comme cela apparaitt sous (25d). L'autre option est de mettre en cause la présence de l'élément A dans les dentales : il existe des propositions qui vont dans ce sens, au moins pour les occlusives $t, d$ (Backley 2011 : 97 discute cette option; voir aussi Scheer 1999). 
(30) a. [p'etə] 'caillou'

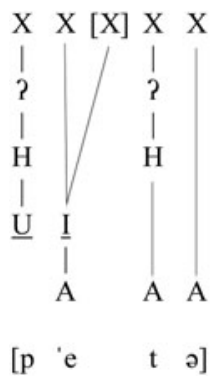

b. [pətr'sttə] 'caillou-DIM'

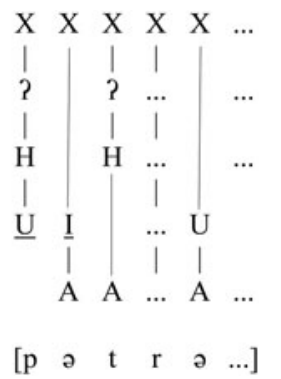

\section{GÉNÉRALISATIONS ET CONCLUSION}

L'objectif de cet article était de rendre compte de l'alternance des voyelles moyennes en coratin en se fondant sur l'analyse de la réduction/du maintien vocalique de ce dialecte proposé dans Bucci (2013a, 2013b) où les voyelles phonologiquement brèves sont réduites à schwa (une seule position syllabique) alors que les voyelles phonologiquement longues sont réalisées pleines. Elles peuvent brancher soit sur l'espace syllabique apporté par l'accent, soit, lorsqu'elles sont atones, sur la mélodie de la consonne adjacente si les deux segments partagent une primitive mélodique.

Cependant, cette première analyse ne permettait pas de rendre compte des alternances des voyelles moyennes en coratin. Les nouveaux ingrédients théoriques (la TE de Backley 2011 et les contraintes de licenciement) permettent maintenant d'affiner l'analyse proposée précédemment et de rendre compte des alternances des voyelles moyennes de ce parler en expliquant pourquoi les voyelles $/ \supset, \varepsilon /$ se réalisent $[\mathrm{o}, \mathrm{e}]$ en position atone lorsqu'elles sont précédées par une consonne labiale pour la première et palatale pour la seconde. Dans ce cadre général, les deux contraintes de licenciement rendent compte, ensuite, de l'alternance de hauteur de /o,o/ qui se réalisent $[\mathrm{u}]$ en position atone lorsqu'elles sont adjacentes à une consonne vélaire. Il semblerait possible d'exporter cette analyse à d'autres dialectes de l'aire italo-romane méridionale des Pouilles orientales. En revanche, les contraintes de licenciement proposées sous (25) diffèreraient pour rendre compte d'autres alternances vocaliques.

\section{RÉFÉRENCES}

Abete, Giovani. 2005. Sulla questione della sillaba superpesante : i dittonghi discendenti in sillaba chiusa nel dialetto di Pozzuoli [Sur la question de la syllabe super lourde : les diphtongues descendantes en syllabe fermée dans le dialecte de Pozzuoli]. Dans Analisi prosodica. Teorie, modelli, sistemi di annotazione. Atti del II Convegno Nazionale dell'Associazione Italiana di Scienze della Voce (AISV) [Analyse prosodique. Théories, modèles, systèmes d'annotation. Actes du Ile congrès national de l'Association italienne des sciences de la voix], sous la direction de Renata Savy et Claudia Renata, Salerno 30 : 379-398. 
Abete, Giovani. 2006. Il polimorfismo delle realizzazioni vocaliche nel dialetto di Pozzuoli [Le polymorphisme des réalisations vocaliques dans le dialecte de Pozzuoli]. Bollettino Linguistico Campano, 9(10) : 143-172.

Anderson, John et Charles Jones. 1974. Three theses concerning phonological representations. Journal of Linguistics 10(1) : 1-26.

Avolio, Francesco. 1995. Bommèspra. Profilo linguistico dell'Italia centro-meridionale [Bommèspra. Profil linguistique de l'Italie centre-méridionale]. San Severo : Gerni.

Archangeli, Diana. 1988. Aspects of underspecification theory. Phonology 5(2) : 183-207.

Backley, Phillip. 2011. An introduction to Element Theory. Edinburgh : Edinburgh University Press.

Bendjaballah, Sabrina. 1995. Aspects apophoniques de la vocalisation du verbe berbère (Kabyle). Dans Actes du $2^{e}$ colloque Langues et Grammaire, sous la direction de Anne Zribi-Hertz, 33-47. Paris : Université Paris 8.

Bendjaballah, Sabrina. 2001. The negative preterite in Kabyle Berber. Folia Linguistica 34(3-4) : 185-223.

Bendjaballah, Sabrina. 2005. Longueur phonologique des voyelles en kabyle. Études et documents berbères 22 : 47-69.

Bertinetto, Pier Marco. 1981. Strutture prosodiche dell'italiano : accento, quantità, sillaba, giuntura, fondamenti metrici [Structure prosodique de l'italien : accent, quantité, syllabe, frontière, fondements métriques]. Firenze : Accademia della Crusca.

Bourciez, Edouard. 1930. Éléments de linguistique romane. Paris : Klincksieck.

Bucci, Cataldo. 1982. Dizionario etimologico coratino [Dictionnaire étymologique du Coratin]. Bari : Tipografica Meridionale.

Bucci, Jonathan. 2013a. Raddoppiamento fonosintattico induit par l'accent et réduction vocalique en Italie : perspectives phonologique et dialectologique. Thèse de doctorat, Université de Nice.

Bucci, Jonathan. 2013b. Voyelles longues virtuelles et réduction vocalique en coratin. Revue canadienne de linguistique 58(3) : 397-414.

Calabrese, Andrea. 2000. The feature [advanced tongue root] and vowel fronting in Romance. Dans Phonological theory and the dialects of Italy, sous la direction de Lori Repetti, 5788. Amsterdam : John Benjamin.

Charette, Monique et Asli Göksel. 1994. Vowel harmony and switching in Turkic languages. SOAS Working Papers in Linguistics and Phonetics 4 : 31-52.

Charette, Monique et Asli Göksel. 1996. Licensing constraints and vowel harmony in Turkic languages. SOAS Working Papers in Linguistics and Phonetics 6 : 1-25.

Chierchia, Gennaro. 1986. Length, syllabification and the phonological cycle in Italian. Journal of Italian Linguistics 8 : 5-34.

Cyran, Eugeniusz. 2012. Cracow sandhi voicing is neither phonological nor phonetic. It is both phonological and phonetic. Dans Sound structure and sense : Studies in memory of Edmund Gussmann, sous la direction de Eugeniusz Cyran, Henryk Kardela, et Bogdan Szymanek. Berlin : Mouton de Gruyter.

D'Imperio, Mariapaola et Sam Rosenthall. 1999. Phonetics and phonology of main stress in Italian. Phonology $16: 1-28$.

D'Introno, Francesco et Rose Mary Weston. 1997. Preservazione, cambio e riduzione vocalica in Coratino : Effetti e strategia dell'OCP [Préservation, changement et réduction vocalique en coratin : Effet et stratégie de l'OCP]. Dans Atti del terzo convegno della Società internazionale di linguistica e filologia italiana [Actes de la troisième conférence de la Société internationale de linguistique et philologie italienne], sous la direction de Luciano Agostiniani, Paola Bonucci, Giulio Gianecchini, Franco Lorenzi et Luisella Reali, 287302. Napoli : Edizioni scientifiche Italiane. 
D'Introno, Francesco et Rose Mary Weston. 2000. Vowel alternation, vowel consonant assimilation and OCP effects in a Barese dialect. Dans Phonological theory and the dialects of Italy, sous la direction de Lori Repetti, 89-110. Amsterdam : John Benjamin.

Fava, Elisabetta et Elisabetta Magno Caldognetto. 1976. Studio sperimentale delle caratteristiche elettroacustiche delle vocali toniche ed atone in bisillabi italiani [Etude expérimentale des caractéristiques électro-acoustiques des voyelles toniques et atones dans les bi-syllabiques en italien]. Dans Studi di fonetica e fonologia [Études de phonétique et phonologie], sous la direction de Raffaele Simone, Ugo Vignuzzi et Giulianella Ruggiero, 35-79. Rome : Bulzoni.

Giammarco, Ernesto. 1973. Abruzzo dialettale [Abruzzo dialectal]. Pescara : Ferretti.

Grassi, Corrado, Alberto Sobrero et Tullio Telmon. 2003. Introduzione alla dialettologia italiana [Introduction à la dialectologie italienne]. Roma-Bari : Laterza.

Gussmann, Edmund. 2002. Phonology, analysis and theory. Cambridge : Cambridge University Press.

Gussmann, Edmund. 2007. The phonology of Polish. Oxford : Oxford University Press.

Harris, John. 1994. English sound structure. Oxford : Blackwell.

Harris, John et Lindsey, Geoff. 1995. The elements of phonological representation. Dans Frontiers of phonology: atoms, structures, derivations, sous la direction de Jacques Durand et Francis Katamba, 34-79. Harlow : Longman.

Honeybone, Patrick. 2005. Sharing makes us stronger : process inhibition and segmental structure. Dans Headhood, elements, specification and contrastivity : Phonological papers in honour of John Anderson, sous la direction de Philip Carr, Jacques Durand et Colin J. Ewin, 167-192. Amsterdam : John Benjamins.

Krämer, Martin. 2009. The phonology of Italian. Oxford : Oxford University Press.

Kaye, Jonathan. 2001. Working with licensing constraints. Dans Constraints and preferences, sous la direction de Katarzyna Dziubalska-Kołaczyk, 251-268. Berlin et New York : Mouton de Gruyter.

Kaye, Jonathan, Jean Lowenstamm et Jean-Roger Vergnaud. 1985. The internal structure of phonological elements : A theory of charm and government. Phonology Yearbook 2 : 305-328.

Larsen, Bergeton Uffe. 1998. Vowel length, raddoppiamento sintattico and the selection of the definite article in Italian. Dans Langues et grammaire, vols. II-III : Phonologie, sous la direction de Patrick Sauzet, 87-102. Paris : Université Paris 8.

Loporcaro, Michele. 1988. Grammatica storica del dialetto di Altamura [Grammaire historique du dialecte d'Altamura]. Pisa : Giardini.

Loporcaro, Michele. 2001. Distinctive features and phonological change : vowel fronting and gravity interactions in Altamurano. Rivista di Linguistica 13 : 255-308.

Loporcaro, Michele. 2011. Syllable, segment and prosody. Dans The Cambridge history of the Romance languages, vol. 1, Structures, sous la direction de Martin Maiden, John Charles Smith et Adam Ledgeway, 50-108, Cambridge : CUP.

Lowenstamm, Jean. 1991. Vocalic length and syllable structure in Semitic. Dans Semitic studies in honor of Wolf Leslau on the occasion of his 85th birthday, sous la direction de Alan S. Kaye, 949-965. Wiesbaden : Harrassowitz.

Melillo, Michele. 1955. Atlante fonetico pugliese [Atlas phonétique des Pouilles]. Roma : Capitanata e Terra di Bari.

Mester, Armin. 1986. Studies in tier structure. Doctoral dissertation, University of Massachusetts. Amherst.

Rohlfs, Gerhard. 1966-1969. Grammatica storica della lingua italiana e dei suoi dialetti [Grammaire historique de la langue italienne et de ses dialectes]. Torino : G. Einaudi. 
Romano, Antonio. 2012a. Frangimenti vocalici coratini : analisi fonetica strumentale con possibilità di rianalisi fonologico-lessicale e contributo alla fonetica storica [Rupture vocaliques du coratin : analyse phonétique instrumentale avec la possibilité d'une nouvelle analyse phonologico-lexicale et contribution à la phonétique historique]. Dans Filologia e linguistica. Studi in onore di Anna Cornagliotti [Philologie et linguistique. Études en honneur à Anna Cornagliotti], sous la direction de Luca Bellone, G. Cura Curà, Mauro Cursietti et Matteo Milani, 877-908. Alessandria : Edizioni dell'Orso.

Romano, Antonio. 2012b. Frangimenti vocalici coratini: difficoltà di rappresentazione e possibilità di analisi strutturale [Rupture vocaliques en coratin : difficulté de représenter et possibilité d'analyse structurelle]. Dans Dialetti: per parlare e parlarne, Atti del secondo Convegno Internazionale di Dialettologia - Progetto A.L.Ba [Dialectes : pour parler et parlant. Actes du second congrès International de dialectologie - Projet A. LBa]. (Potenza-Venosa-Matera 13-15 maggio 2010), sous la direction de Patrizia Del Puente, 239-262. Rionero in Vulture (PZ) : Calice Editori 2011.

Russo, Michela. 2010. Le origini della dittongazione spontanea nei dialetti italiani meridionali dell'ovest (Ischia e Pozzuoli) : isocronia diacronica antischürriana e quantificazioni isocroniche attuali della situazione arcaica [Les origines de la diphtongue spontanée dans les dialectes italiens méridionales de l'ouest (Ischia e Pozzuolli) : isochronie diachronique antischürriana et quantifications isochroniques actuelles dans la situation archaïque]. Zeitschrift für romanische Philologie (ZrP) 126(2) : 304-349.

Savoia, Leonardo M. 1987. Teoria generativa, modelli fonologici e dialettologia. La propagazione di $u$ in una varietà lucana [Théorie générative, modèles phonologiques et dialectologie. La propagation de $u$ dans une variété lucane]. Rivista italiana di dialettologia $11: 185-263$.

Savoia, Leonardo M. 1990. Proprietà vocaliche e struttura metrico-sillabica in alcune varietà abruzzesi. [Propriété vocalique et structure métrico-syllabique dans certaines variétés des Abruzzes] Dans Studi in memoria di Ernesto Giammarco [Etudes en mémoire d'Ernesto Giammarco], 331-380. Pisa : Giardini.

Savoia, Leonardo M. 2015. I dialetti italiani. Sistemi e processi fonologici nelle varietà di area italiana e romancia [Les dialectes italiens. Systèmes et processus phonologiques dans les variétés de l'aire italienne et romane]. Pisa : Pacini.

Savoia, Leonardo M. et Elisabetta Carpitelli. 2008. Problèmes de micro-variation phonologique dans les domaines dialectaux de l'Italie septentrionale. Revue française de linguistique appliquée 13(2) : 103-119.

Scheer, Tobias. 1999. A theory of consonantal interaction. Folia Linguistica 32 : 201-237.

Scheer, Tobias. 2010. How to marry (structuralist) contrast and (generative) processing : Review of Dresher 2009, The contrastive hierarchy in phonology. Lingua 120 : 25222534.

Ségéral, Philippe et Tobias Scheer. 2008. The Coda mirror, stress and positional parameters. Dans Lenition and fortition, sous la direction de Joaquim Brandão de Carvalho, Tobias Scheer et Philippe Ségéral, 235-271. Berlin : Mouton de Gruyter.

Stehl, Thomas. 1980. Die Mundarten Apuliens : historische und strukturelle Beiträge [Les dialectes des Pouilles : contributions historiques et structurelles]. Münster Westfalen : Aschendorff.

Stehl, Thomas. 1988. Italienisch: Areallinguistik XI : Apulien und Salento. [Italien : Linguistique régionale XI : Puglia et Salento] Sprachen und Sprachgebiete 6 : 696-716.

Valente, Vincenzo. 1975. Profilo dei dialetti italiani : Puglia [Profil des dialectes italiens : Pouilles]. Pisa : Pacini.

Vogel, Irene. 1982. La sillaba come unità fonologica [La syllabe comme unité phonologique]. Bologna : Zanichelli. 